\title{
Identification of protein biomarkers in nipple aspiration fluid
} S Krishnamurthy

\author{
Address: MD Anderson Cancer Center, Houston, Texas, USA
}

Email: S Krishnamurthy - skrishna@mdanderson.org

from 6th International Symposium on the Intraductal Approach to Breast Cancer Santa Monica, CA, USA. 19-2I February 2009

Published: 24 July 2009

BMC Proceedings 2009, 3(Suppl 5):S7 doi:10.1 |86/1753-656|-3-S5-S7

This abstract is available from: http://www.biomedcentral.com/1753-656I/3/S5/S7

(c) 2009 Krishnamurthy; licensee BioMed Central Ltd.

Nipple aspiration fluid (NAF) can be useful for studying relevant changes in breast parenchyma and identification of potential biomarkers for detection, monitoring therapy and risk assessment of breast cancer. NAF has been investigated extensively for identification of protein biomarkers using approaches such as ELISA, immunoassay, western blot for chosen individual protein markers such as prostate specific antigen, basic fibroblast growth factor, prostaglandin E2 etc., or by using comprehensive analysis of protein expression using platforms such as surface enhanced laser desorption ionization-time of flight mass spectrometry. The results of the former studies indicate the potential value of selected markers for detection of cancer which have not been validated in larger studies. The results of majority of studies that performed comprehensive protein analysis indicate that protein expression patterns are highly conserved between cancerous and non cancerous breasts and that protein profiling of NAF may have more value for risk assessment than for detection of breast cancer. Using a novel approach of protein microarray for seven markers with significant differences in protein levels were found between NAF obtained from cancerous and contralateral breast and healthy controls for markers such as cathepsin and urokinase. Overall, proteomic studies of NAF indicate the probable value of several protein biomarkers. There is a need for validation of the markers that have been identified so far, to be promising for risk assessment or early detection of breast cancer in large prospective clinical trials. 